\title{
Screening and function discussion of a hereditary renal tubular acidosis family pathogenic gene
}

\author{
Li Chen ${ }^{1,2}$, Han-Lu Wang', Yao-Bin Zhu', Zhao Jin', Jian-Bin Huang ${ }^{1}$, Xin-Fu Lin ${ }^{1,4}$, Jie-Wei Luo $\mathbb{0}^{1,2}$ and \\ Zhu-Ting Fang ${ }^{1,5}$
}

\begin{abstract}
Hereditary distal renal tubular acidosis (dRTA) is a rare disease of $\mathrm{H}^{+}$excretion defect of a-intercalated cells in renal collecting duct, caused by decreased V-ATPase function due to mutations in the ATP6V1B1 or ATP6VOA4 genes. In the present study, a genetic family with 5 members of the complete dRTA phenotype were found with distal tubule $\mathrm{H}^{+}$ secretion disorder, hypokalemia, osteoporosis, and kidney stones. A variant NM_020632.2:c.1631C > T (p.Ser544Leu) in exon 16 on an ATP6VOA4 gene associated with dRTA was detected by next generation sequencing target region capture technique and verified by Sanger sequencing, which suggested that except for one of the patients who did not receive the test, the other four patients all carried the p.S544L heterozygote. In transfected HEK293T cells, cells carrying p.S544L-mut showed early weaker ATPase activity and a slower Phi recovery rate after rapid acidification. By immunofluorescence localization, it was observed that the expression level of p.S544L-mut on the cell membrane increased and the distribution was uneven. Co-immunoprecipitation showed the a4 subunit of ATP6VOA4/P.S544L-mut could not bind to the B1 subunit, which might affect the correct assembly of V-ATPase. The present study of dRTA family suggests that the p.S544L variant may be inherited in a dominant manner.
\end{abstract}

\section{Introduction}

Renal tubular acidosis (RTA) is a clinical syndrome characterized by normal anion gap (AG) and high chloride metabolic acidosis caused by renal acidification dysfunction. It could be caused by $\mathrm{H}^{+}$obstruction of distal renal tubules, or proximal renal tubules for bicarbonate $\left(\mathrm{HCO}_{3}{ }^{-}\right)$reabsorption, or both. Clinically, it is divided into four types: distal RTA (type I, dRTA), proximal RTA (type II, pRTA), mixed RTA (type III), hyperkalemic RTA (type IV $)^{1-3}$. The etiologies of RTA might be primary tubular disease, systemic disease, or side effects of

Correspondence: Jie-Wei Luo (docluo0421@aliyun.com) or

Zhu-Ting Fang (470389481@qq.com)

${ }^{1}$ Shengli Clinical Medical College of Fujian Medical University, Fuzhou 350001,

China

2Department of Traditional Chinese Medicine, Fujian Provincial Hospital,

Fuzhou 350001, China

Full list of author information is available at the end of the article.

These authors contributed equally: Li Chen, Han-Lu Wang, Yao-Bin Zhu,

Zhao Jin

Edited by S. Kumar medicine. Most of the primary defects are congenital defects of the renal tubules and often related to heredity; secondary diseases could be seen in many diseases, mainly are autoimmune diseases such as Sjogren's syndrome, systemic lupus erythematosus ${ }^{3-7}$. dRTA is defined as the disease caused by distal renal tubular $\mathrm{H}^{+}$secretion disorder, reduced discharge of urine $\mathrm{NH}_{4}{ }^{+}$and titratable acid. Urine acidification dysfunction means that the $\mathrm{pH}$ could not be reduced to 5.3-5.5 in the case of systemic metabolic acidosis, which is clinically common $^{7,8}$. Hereditary dRTA is a rare disease caused by genetic mutation resulting in $\mathrm{H}^{+}$excretion defect of $\alpha$-intercalated cells ( $\alpha$ ICs) in renal collecting duct. Its main clinical features are persistent normal AG hyperchloric metabolic acidosis, hypokalemia, osteoporosis, and renal calcinosis ${ }^{9}$. The present study describes a clinical phenotype and genetic responsibility variant screening for a dRTA family, followed by functional identification.

\section{(c) The Author(s) 2020}

(c) (i) Open Access This article is licensed under a Creative Commons Attribution 4.0 International License, which permits use, sharing, adaptation, distribution and reproduction cc) in any medium or format, as long as you give appropriate credit to the original author(s) and the source, provide a link to the Creative Commons license, and indicate if changes were made. The images or other third party material in this article are included in the article's Creative Commons license, unless indicated otherwise in a credit line to the material. If material is not included in the article's Creative Commons license and your intended use is not permitted by statutory regulation or exceeds the permitted use, you will need to obtain permission directly from the copyright holder. To view a copy of this license, visit http://creativecommons.org/licenses/by/4.0/. 


\section{Materials and methods}

\section{Research objects}

Proband (III12, Fig. 1a): male, 19 years old, Chinese Han, chief complaint was "recurring generalized fatigue for 5 years", and denied numbness of limbs, convulsions, urine volume abnormality, abnormal development, and hearing loss. The serum potassium level was 2.2-2.8 mmol/L checked at local hospital, normal blood pressure, symptoms could be alleviated after oral potassium chloride treatment, serum potassium could raise to the normal range when reexamined. Two years before admitted to the hospital, the patient often felt pain in the right heel after standing for a long time. Magnetic resonance imaging of the right calcaneus (Fig. 1b) examined in the local hospital showed a small amount of fluid in right ankle joint, bursitis and Achilles tendinitis in right ankle anterior Achilles tendon. The patient felt general weakness three days before admission. Serum potassium was $2.8 \mathrm{mmol} / \mathrm{L}$ and chlorine was $112 \mathrm{mmol} / \mathrm{L}$ examined in the local hospital. Symptoms were relieved after intravenous infusion of potassium chloride. For further diagnosis and treatment, the patient was referred to our hospital. The patient was the first child of the first-time pregnancy and denied that his parents were close relatives. After admission, a series of examinations such as electrocardiogram, chest and pelvic radiographs, and arterial blood gas analysis were performed on the proband. A family survey was conducted and a hereditary pedigree was made (Fig. 1a), finding five family members with similar medical history. Ammonium chloride $\left(\mathrm{NH}_{4} \mathrm{Cl}\right)$ loading test was performed on four patients. The study was approved by the Ethics Committee of the Fujian Provincial Hospital and all members of the family surveyed signed an informed consent form.

\section{Candidate gene mapping and mutation screening strategy}

Peripheral blood of the proband and a part of family members were collected with an ethylenediaminetetraacetic acid anticoagulant tube. The peripheral blood DNA was extracted according to the instructions of QIAamp DNA Blood Mini Kit (QIAGEN, Cat No. 51106) and purified for subsequent experiment. The purity of the extracted DNA was determined using a NanoDrop instrument. The OD260/OD280 ratio was maintained at 1.8-2.0 to meet the subsequent sequencing process. At first, DNA whole genome library preparation, capture of target gene regions was performed. Next, sequencing was performed using a sequencing panel on the Illumina NextSeq 500 platform. This panel could be used to perform parallel analysis of multiple genes. The target genes involved are: SLC4A1, ATP6VOA4, CA2, SLC4A4, and ATP6V1B1. The sequencing data including all the SNVs and small indels were analyzed using ANNOVAR software $^{10}$, and several databases, including 1000G, ESP6500,
dbSNP, and HGMD were used according to the ACMG guidelines $^{11}$ to filter and annotate variants. The reads obtained from were filtered, separated, and BWA and hg19 sequences were used for reference aligned to acknowledge important information about the variants, such as gene locus, variant type, 1000G and ESP6500 frequencies. Sorting intolerant from tolerant (SIFT, http:// sift.jcvi.org/), polymorphism phenotyping (PolyPhen-2, http://genetics.bwh.harvard.edu/ppH2/), and mutation taster (http://mutationtaster.org/) were used to predict the pathogenicity of the disease to obtain gene mutation information and its biological significance. Primers 5.0 were used to design primers for the position of the suspect variant site (http://sg.idtDNA.com/Primerquest/ Home/Index) and the target region was amplified. Sanger sequencing was performed on the ABI $3500 \mathrm{Dx}$ platform to verify Illumina NextSeq 500 sequencing results. For example, the polymerase chain reaction (PCR) amplified fragment of the target sequence of NM_020632.2:c.1631C > T (p.Ser544Leu) of the suspicious variant $A T P 6 V 0 A 4$ was $274 \mathrm{bp}$, the primer was F: CCAAACCAGTGGCTCTGTCA; R: GTTGTGCTGTA GCCCTCAACT, and the annealing temperature was $62{ }^{\circ} \mathrm{C}$. The primers were synthesized by Suzhou Synbio Technology Co., Ltd.

\section{Construction and transfection of ATP6VOA4 gene p.S544L wild and mutant eukaryotic plasmids}

The plasmids construction of the fragment in which $\mathrm{p}$. S544L is located was constructed by gene synthesis. Primers were designed according to this principle: each primer must carry the desired variant site and the designed variant site should be located in the center of the primer. Primers: A4 Mut-F: CTCGTATAAAATGAAG ATGTTGGTGATCCTGGGAATTGTCC; A4 Mut-R: G GACAATTCCCAGGATCACCAACATCTTCATTTTATACGAG; high-fidelity primer star polymerase was used and followed by 18 cycles of PCR reaction. EcoRI and BamHI in the vector plasmid pEGFP-Nl were selected as the restriction sites. After PCR purification, the target fragments were ligated with pJet1.2 vector (Xinyu, Shanghai, China) by using T4 DNA ligase (Thermo Fisher) to obtain a large number of intermediate plasmids of the desired fragment. The competent Escherichia coli cell DH5a strain was prepared through the $\mathrm{CaCl}_{2}$ method to express foreign genes. After the enzyme ligated products were transformed into the competent $E$. coli DH5a strain, then E. coli was coated on a medium containing the corresponding antibiotic to select the mutant type. If the foreign plasmid DNA is successfully transformed into $E$. coli, it could be grown on a medium containing an antibiotic (ampicillin sodium, Shanghai Biotech, China). Afterwards, a large number of recombinant plasmids were cloned by expanding the culturation of E. coli. A small 


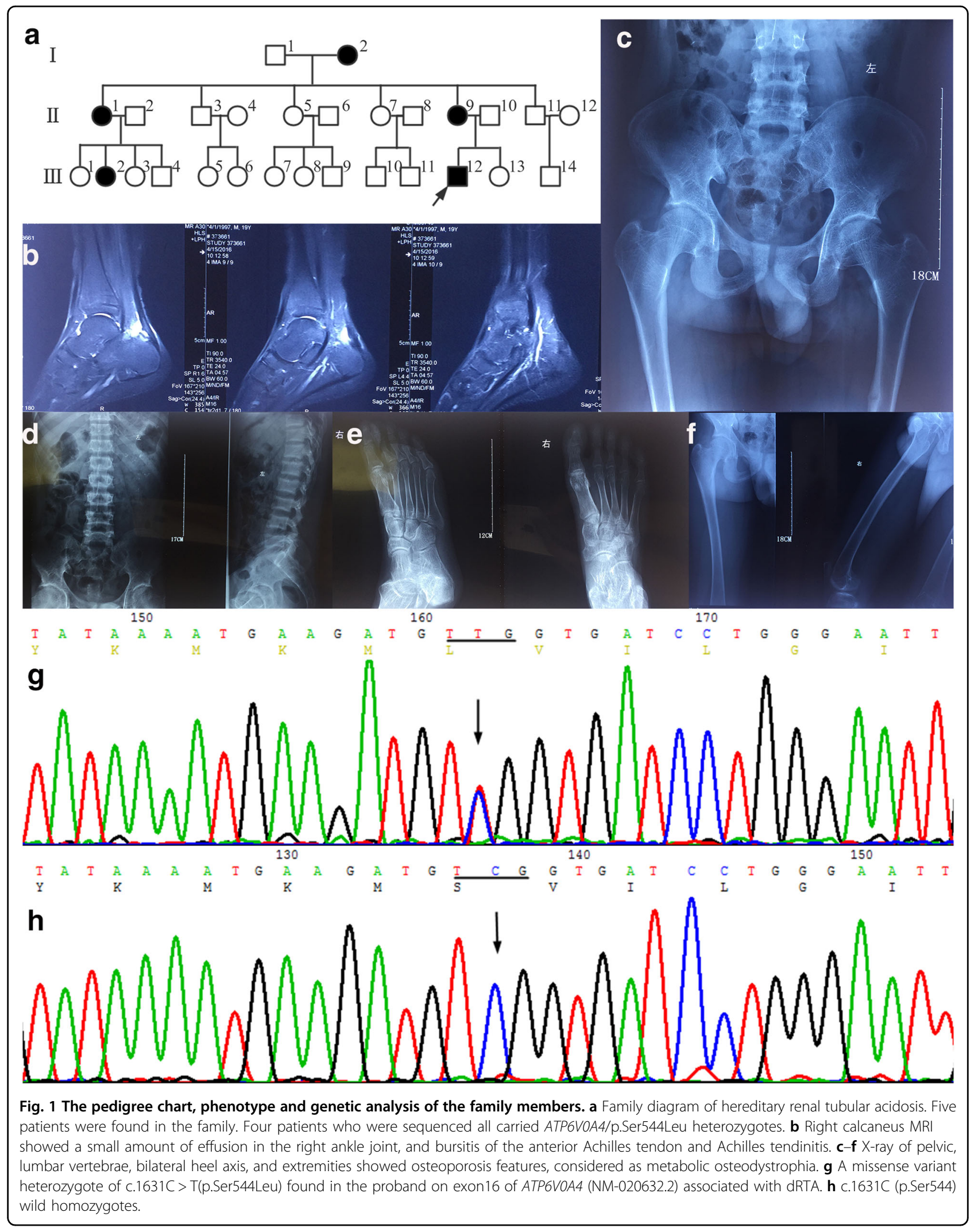


amount of plasmid DNA was extracted and verified by sequencing. HEK293T cells were cultured in DMEM medium with high glucose $+10 \%$ fetal bovine serum $+1 \%$ $\mathrm{P} / \mathrm{S}$ (Hyclone medium, SH30022.01B), and transfected with plasmids (TurboFect transfection reagent, Thermo, R0531). Total RNAs were extracted from the cultured cells according to the instructions of the Roche reagent kit Tripure isolation reagent (ROCHE, 11667165001). In addition, after the $A T P 6 V 1 B 1$ overexpression plasmid was biosynthesized, the CDS region of $A T P 6 V 1 B 1$ was constructed into the pBobi-N-3HA vector.

\section{Protein extraction and western blot analysis}

A $20 \mathrm{mg}$ of cell tissue was lysed by RAPI lysate to extract total protein. The protein concentration was determined by the bicinchoninic acid assay (BCA) method (BCA Protein Quantification Kit, Biyuntian, P0011), the absorbance at $562 \mathrm{~nm}$ was measured, and the protein concentration of the sample was calculated according to the standard curve. Equal amounts of protein were loaded per lane using $10 \%$ sodium dodecyl sulfate (SDS)-polyacrylamide gel electrophoresis, transferred onto polyvinylidene fluoride membranes and blocked for $1 \mathrm{~h}$ at room temperature in 5\% skim milk prepared in TBST. The membrane was cut as needed and immersed in the prepared primary antibody solution at the dilutions recommended by the manufacturer, incubated overnight at $4{ }^{\circ} \mathrm{C}$. Next, the membrane was incubated with secondary antibody which was selected according to primary antibody and diluted at 1:5000 at room temperature for $1 \mathrm{~h}$, and ECL reagent was added to visualize the immunostained proteins.

\section{Immunofluorescence localization}

The fixed cells were stored in phosphate-buffered saline (PBS) containing Sodium azide at $4{ }^{\circ} \mathrm{C}$ for 3 months. After washing with PBS, the cells were blocked using blocking solution for $30 \mathrm{~min}$. Anti-flag (1:500, sigma, F2555) primary antibody and anti-Ms-488 (1:1000, Jackson, 209545-082) secondary antibody were added into the cells, respectively. The nuclei were stained with DAPI and incubated for $1 \mathrm{~h}$ in the dark. High-sensitivity laser confocal microscopy (Zeiss, LSM780) was used to observe the cells after mounting.

\section{Co-immunoprecipitation (Co-IP)}

Co-IP was performed with HA antibody and Flag antibody respectively, and 5\% input sample was detected using tubulin as an internal reference and green fluorescent protein (GFP) as an external reference. After $10 \mu \mathrm{g}$ of the plasmids were transfected by groups, $2 \mu \mathrm{g}$ of the GFP control plasmids were added to $1000 \mu \mathrm{L}$ of OptiMedium and mixed into a TurboFect-DNA mixture.
Afterwards, $20 \mu \mathrm{L}$ of TurboFect was added and the mixed solutions was added dropwise to a single layer of HEK293T cells. After $48 \mathrm{~h}$ of transfection, the cells were harvested, lysed on ice, centrifuged at $4{ }^{\circ} \mathrm{C}, 15,000 \mathrm{~g}$ for $15 \mathrm{~min}$, and the supernatant was stored at $-20^{\circ} \mathrm{C}$. Totally, $5 \mu \mathrm{L}$ of whole-cell lysates was taken as an input (5\%). Totally, $100 \mu \mathrm{L}$ protein samples were, respectively, added with $1 \mu \mathrm{L} \mathrm{HA}$ antibody, Flag antibody and $5 \mu \mathrm{L}$ protein A/ $\mathrm{G}$ magnetic beads for reaction overnight at $4{ }^{\circ} \mathrm{C}$. The Ep tube was placed on the magnetic stand, the supernatant was removed and $1 \mathrm{~mL}$ of protein lysate was added. The mixed solution was shaken and placed on the mixer for 5 min which was repeated for 4 times. The sample was prepared by adding $30 \mu \mathrm{L}$ of $1 \times$ SDS loading buffer into solution, and the Western Blot analysis was performed along with the input. Primary anti-mouse Monoclonal ANTI-FLAG ${ }^{\circ}$ M2 antibody (SIGMA, F3165), Anti-HA tag (Abcam, ab9110), mouse monoclonal anti-GFP antibody (SIGMA, SAB-2702197), tubulin antibody (Abcam, ab7291) were diluted with the proportion of 1:1000, 1:1000, 1:1000, 1:2000 with blocking solution. The secondary anti-Goat anti Mouse IgG (Thermo, 31430) was diluted with the proportion of 1:2000 and incubated for $1 \mathrm{~h}$ at room temperature on a shaker.

\section{Determination of ATPase activity and ATPase hydrogen ion transport function in transfected cells}

The hydrolysis of ATPase producing ADP is accompanied by oxidation reaction of reduced nicotinamide adenine dinucleotide (NADH). Therefore, the unit enzyme activity could be measured by determining the change in the absorbance peak (NADH concentration) at $340 \mathrm{~nm}$ after oxidation by spectrometry. The coimmunoprecipitate product was mixed with $1 \times$ ATPase reaction buffer for $25 \mathrm{~min}$ and the change in concentration of $\mathrm{NADH}$ was determined. The Vacuolar $\left(\mathrm{H}^{+}\right)$ATPase (V-ATPase) mediated hydrogen ion transport function was determined by fluorescence spectrophotometer (Thermo) method: The HEK293T cells expressing ATPVOA4-WT and ATPVOA4-mut proteins in a single layer were rapidly acidified using $\mathrm{NH}_{4} \mathrm{Cl}$, and the transport function of ATPase hydrogen ions was measured by calculating the recovery rate of $\mathrm{PH}$ of cells that was independent on sodium transport. The research tool of this experiment was a multifunction microplate reader (Thermo, VARIOSKAN LUX).

\section{Statistics}

The experimental data was statistically processed using graphpad Prism 6.02. The mean was expressed as mean \pm SEM. The mean of multiple groups was compared by oneway analysis of variance. $P<0.05$ was considered as statistically significant. 


\section{Results}

\section{Clinical data survey of RTA family}

Proband (III12), male, 19 years old, chronic hypokalemia. The clinical manifestations were hypodynamia and lower hypokalemia under repeated stress, and serum potassium fluctuated at $2.2-2.8 \mathrm{mmol} / \mathrm{L}$. After admission, the biochemical indicators of the probands (Table 1) showed serum potassium $2.74 \mathrm{mmol} / \mathrm{L}$, serum phosphorus $0.66 \mathrm{mmol} / \mathrm{L}$, serum chlorine $110 \mathrm{mmol} / \mathrm{L}$, alkaline phosphatase $329 \mathrm{U} / \mathrm{L}$, normal serum sodium and calcium, suggested hypophosphatemia, hyperchloremia, and hypokalemia. Various tumor markers (AFP, CEA, CA125, CA199, and CA153) are normal. Bone density measurement showed that the bone density of the left forearm was $0.236 \mathrm{~g} / \mathrm{cm}^{2}$, which was five standard deviation lower than the peak bone mass of the same sex and five standard deviation lower than that of the same age, suggesting osteoporosis. X-ray of the frontal position of pelvis, lumbar vertebrae, and lateral position of both heel axes (Fig. 1c-e) showed that reduced bone mineral density, the trabecular bone was sparse, the cortical bone was thickened, blurred, and the density was uneven, the trabecular bone was rough and fuzzy, which might be considered as metabolic bone malnutrition. X-ray of limbs (Fig. 1f) showed that the femur and tibia diaphyses flexion deformities, metaphyses were slightly enlarged, the cortical bone was thin, the trabecular bone was irregular and fuzzy, and several transverse growth barriers were visible in the lower part of the tibia, calcaneus bone spur was formed. $24 \mathrm{~h}$ : urinary potassium $72.2 \mathrm{mmol} / \mathrm{L}$ (reference value $25.0-125.0 \mathrm{mmol} / \mathrm{L}$ ), urinary sodium $127 \mathrm{mmol} / \mathrm{L}$ (reference value $40.0-220.0 \mathrm{mmol} / \mathrm{L}$ ), urinary calcium $6.5 \mathrm{mmol} / \mathrm{L}$ (reference value $2.5-7.5 \mathrm{mmol} / \mathrm{L}$ ), urinary chlorine $124 \mathrm{mmol} / \mathrm{L}$ (reference value $170-250 \mathrm{mmol} / \mathrm{L}$ ). Blood gas analysis showed $\mathrm{PH} 7.333, \mathrm{PaCO}_{2} 36.4 \mathrm{mmHg}$, $\mathrm{PaO}_{2} 94.8 \mathrm{mmHg}$, Hct 55\%, Hb $179 \mathrm{~g} / \mathrm{L}$, extracellular residual base $-6.0 \mathrm{mmol} / \mathrm{L}$, residual base $-5.9 \mathrm{mmol} / \mathrm{L}$, standard bicarbonate (SB) $119.7 \mathrm{mmol} / \mathrm{L}$, actual bicarbonate $(\mathrm{AB}) \mathrm{HCO}_{3}{ }^{-} 18.8 \mathrm{mmol} / \mathrm{L}$, total carbon dioxide $19.9 \mathrm{mmol} / \mathrm{L}$, and lactic acid $2.0 \mathrm{mmol} / \mathrm{L}$. Blood AG $15.2 \mathrm{mmol} / \mathrm{L}$, suggesting metabolic acidosis, the proband (III12) could be diagnosed as RTA. The other four members of the family (Fig. 1a) (I2, II1, II9, and III2) also have different degrees of clinical symptoms such as fatigue, anorexia, kidney stones, or hypokalemia. Detailed clinical data and blood samples of the proband's grandmother (I2) were not collected. Family members had no growth retardation, mental retardation, facial deformity, dental caries, mental illness, and no deafness, eye disease, or congenital heart disease. All the four diseased members including the proband, had different degrees of hypokalemia. After the ammonium chloride load test, the urine pH of II1, II5, III2, and III12 (proband) were greater than 5.3. Therefore, the family was considered as a hereditary
dRTA family. In the ammonium chloride load test, it is a kind of test to check the function of distal renal tubular, urine $\mathrm{pH}>5.3$ after acidification indicates positive, which means that distal renal tubular acidification dysfunction caused type I RTA.

\section{Target region capture high-throughput sequencing and Sanger sequencing verification}

Under the condition of ensuring sufficient coverage and depth of NGS sequencing, the probands of this family were subjected to high-throughput sequencing analysis of 6 gene target regions, including SLC4A1, ATP6VOA4, CA2, SLC4A4, and ATP6V1B1. The exon coding region and flanking regions of target genes were screened in detail, and copy number variation analysis was performed on all target genes. No major deletions or insertions were found in the patient (Table 2), and the mutant polymorphism (single-nucleotide polymorphisms (SNP)) locus with MAF (this value came from the population frequency information about dbSNP from the 1000 genomes project) greater than $1 \%$ was removed by filtration. Compared with the Clinvar database, 11 rare variants were screened, and no large deletions or duplications were found. A ATP6VOA4 gene (NM-020632.2) exon16 variant associated with dRTA was detected, which is a missense variant heterozygote and named c.1631C > T (p. Ser544Leu) according to the HGVS international specification (http://www.hgvs.org/mutnomen/). This variant was not included in the ESP6500, dbSNP, and 1000G databases. Confirmed by Sanger sequencing (Fig. 1g, h), the amino acid at position 544 of exon 16 of the ATP6V0A4 gene was changed from serine (Ser) to leucine (Leu) and the Clinvar database also has no relevant listings for this site (https://www.ncbi.nlm.nih.gov/clinvar/? term $=$ ATP6V0A4\%5Bgene\%5D). Except for one unsampled case (I2), the other four patients (I2, II1, II9, and III2) carried the missense variant of p.S544L, and unaffected II5, III7, II10 (father of the proband), and III13 did not carry this variant. The family genetic linkage analysis showed that this variant had genetic co-segregation phenomenon. The PolyPhen-2 pathogenicity prediction score for the ATP6V0A4/p.S544L variant is 1 and the SIFT prediction score is 0 . The variant is predicted to be harmful, which will cause a conformational change in the encoded protein and affect its normal function. Also Ser residues are often associated with phosphorylation. A mutation from Ser to Leu may affect protein function caused by loss of posttranslational modification ${ }^{12}$.

\section{ATP6V0A4/p.S544L gene mutant clone}

In the present study, ATP6VOA4 wild-type (-WT) and ATP6V0A4/p.S544L cloning vectors and eukaryotic expression vectors were successfully constructed. The structure of some vectors is shown in following Fig. 2a. 


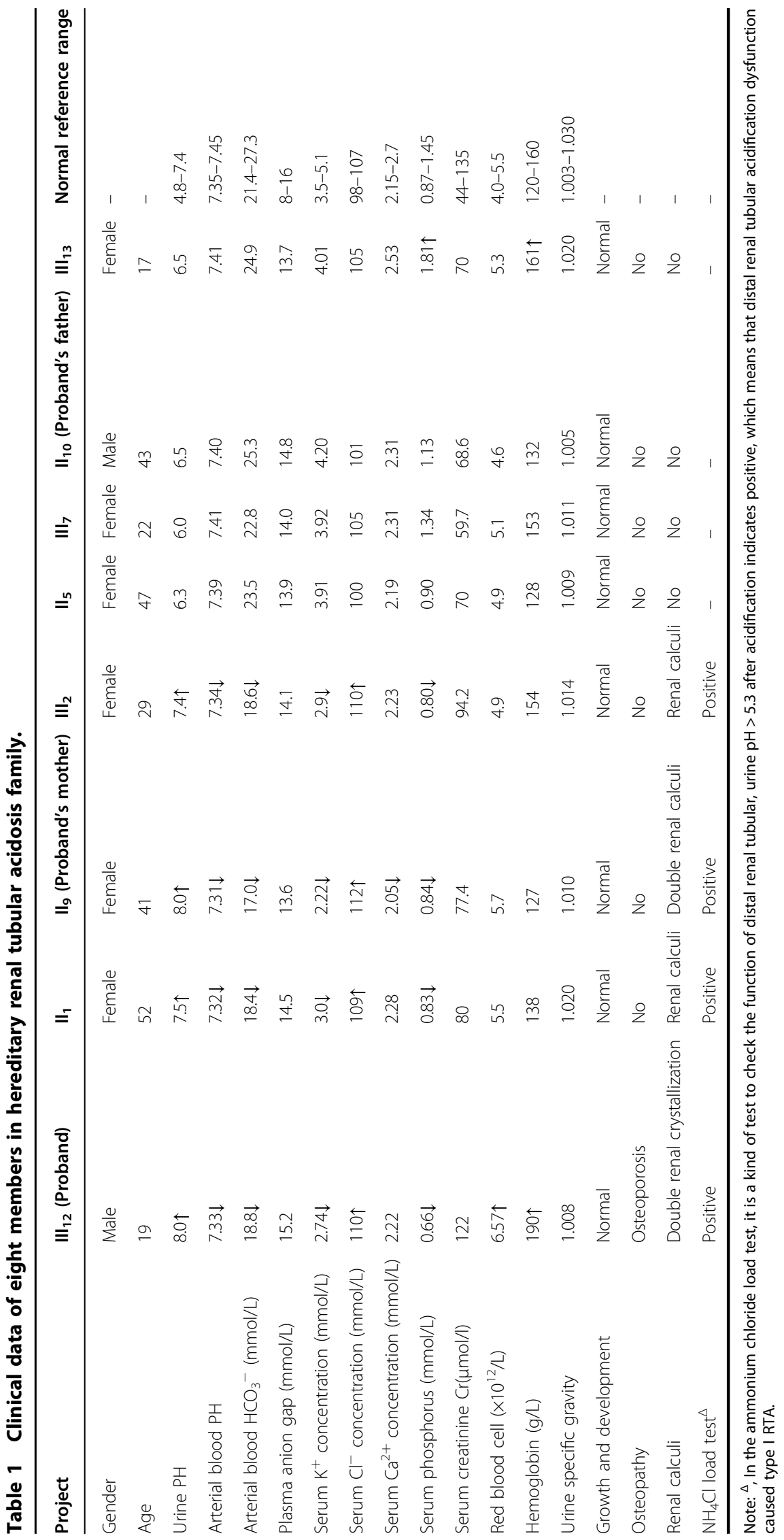


Table 2 Overview of screening for proband-related gene mutations in hereditary renal tubular acidosis family.

\begin{tabular}{|c|c|c|c|c|c|c|c|c|c|}
\hline Gene & RefSeq & $\begin{array}{l}\text { Nucleic acid } \\
\text { alternation }\end{array}$ & $\begin{array}{l}\text { Amino acid } \\
\text { alternation }\end{array}$ & Mut site & Zygotic & $\begin{array}{l}\text { Chromosome } \\
\text { location }\end{array}$ & RS-ID & Maf & Mut type \\
\hline ATP6V0A4 & NM_020632 & c.C1631T & p.S544L & exon16 & Het & chr7 & - & - & Mis \\
\hline ATP6V1B1 & NM_001692 & c.C89T & p.T30l & exon1 & Het & chr2 & rs17720303 & 0.16 & Mis \\
\hline ATP6V1B1 & NM_001692 & c.C138T & p.S46S & exon2 & Het & chr2 & rs2266918 & 0.28 & Syn \\
\hline ATP6V1B1 & NM_001692 & c.C1002T & p.H953H & exon10 & Hom & chr2 & rs2072462 & 0.44 & Syn \\
\hline SLC4A4 & NM_003759 & c.C2859T & p.H604H & exon20 & Hom & chr4 & rs1453458 & 0.78 & Syn \\
\hline ATP6V0A4 & NM_130840 & c.T1812C & p.F554F & exon16 & Hom & chr7 & rs3807154 & 0.68 & Syn \\
\hline ATP6V0A4 & NM_130840 & c.C1662T & p.L87L & exon15 & Hom & chr7 & rs1026435 & 0.72 & Syn \\
\hline ATP6V0A4 & NM_130840 & c.T5C & p.V2A & exon2 & Hom & chr7 & rs10258719 & 0.68 & Mis \\
\hline CA2 & NM_0012936 & c.T259C & p.L87L & exon5 & Het & chr8 & rs703 & 0.58 & Syn \\
\hline SLC4A1 & NM_000342 & c.G1314A & p.S438S & exon12 & Het & chr17 & rs13306781 & 0.01 & Syn \\
\hline SLC4A1 & NM_000342 & C.A166G & p.K56E & exon4 & Het & chr17 & rs5036 & 0.06 & Mis \\
\hline
\end{tabular}

Note: RefSeq NCBI reference sequence, Mutation Mut, Het heterozygote, Hom homozygous, cytoBand the chromosome segment in which the mutation is located, Mis missense mutation, Syn synonymous, Nonfs ins nonframeshift insertion, FRE frequency, MAF SNP minor allele frequency from dbSNP 1000 Genomes (population frequency information from the 1000 genomes project).

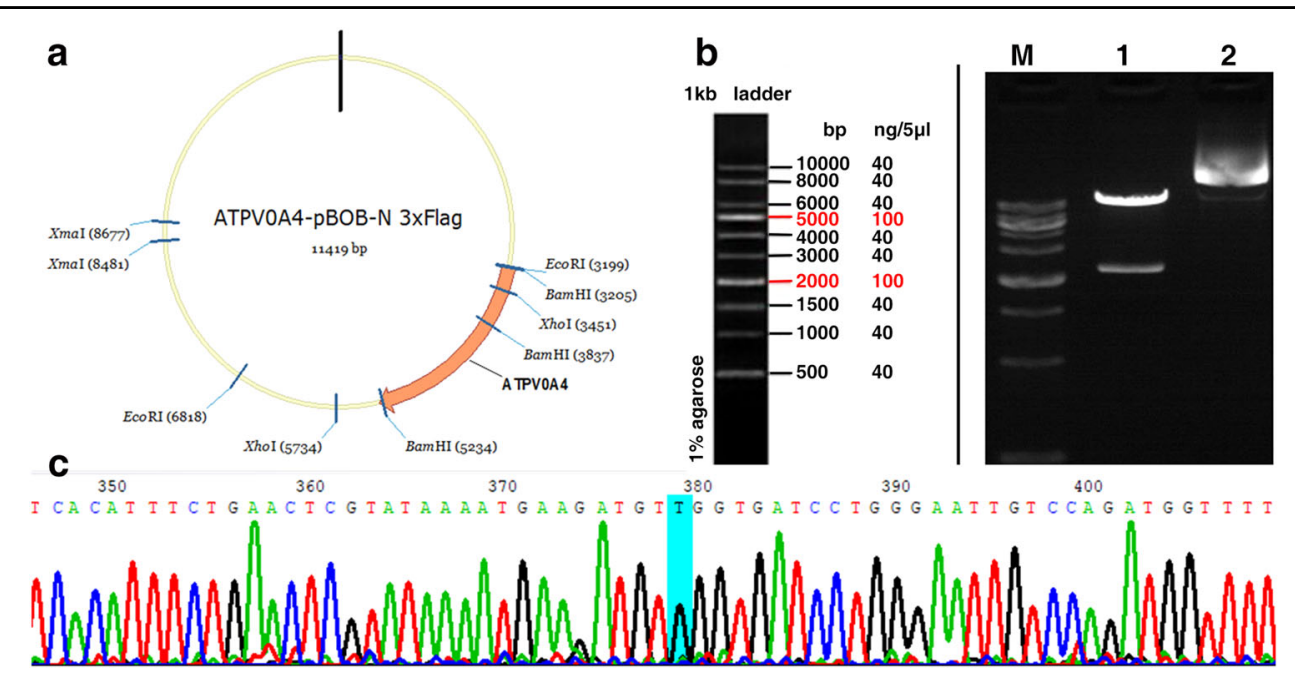

Fig. 2 ATP6V0A4/p.S544L gene cloning. a ATP6V0A4/p.S544L mutant clone, schematic diagram of partial vector. b ATP6V0A4 wild type and ATP6V0A4/p.S544L mutant fragment by Xhol restriction enzyme digestion. c Successfully constructed vector sequenced verification.

The wild-type ATP6VOA4 and the mutant ATP6VOA4/p. S544L (-mut) were digested by Xhol producing fragments approximately at 2000 and $5000 \mathrm{bp}$, which were consistent with the design (Fig. 2b). Successfully constructed vectors were verified by sequencing (Fig. 2c) and successfully transfected into HEK293T cells.

Expression of ATP6VOA4 gene and mutant in HEK293T cells and interaction with B1 subunit

Immunofluorescence showed that both ATP6V0A4-WT and ATP6V0A4/p.S544L-mut proteins could be expressed on the cytoplasm, and most of them were expressed on the cell membrane. Compared with WT, we observed that the expression of ATP6V0A4/p.S544L-mut on the cell membrane increased and the distribution was not uniform, but the morphology of the cells did not change significantly (Fig. 3a). This indicates that the p.S544L-mut variant is retained in the cell membrane, and the mutation may affect the removal of this variant from the cell membrane. V-ATPase is a multi-subunit complex located in the $\mathrm{B} 1$ subunit of the V1 domain of the cytoplasmic VATPase. In the brush border membrane of $\alpha-\mathrm{ICs}$ of the kidney, whether the V-ATPase is correctly assembled with the complete structure of the a4 subunit of the V0 domain 

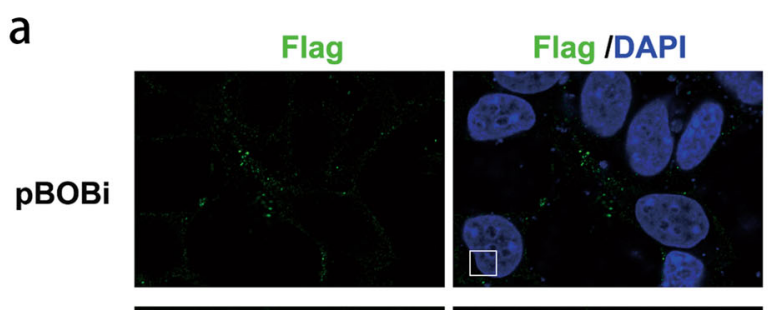

A4
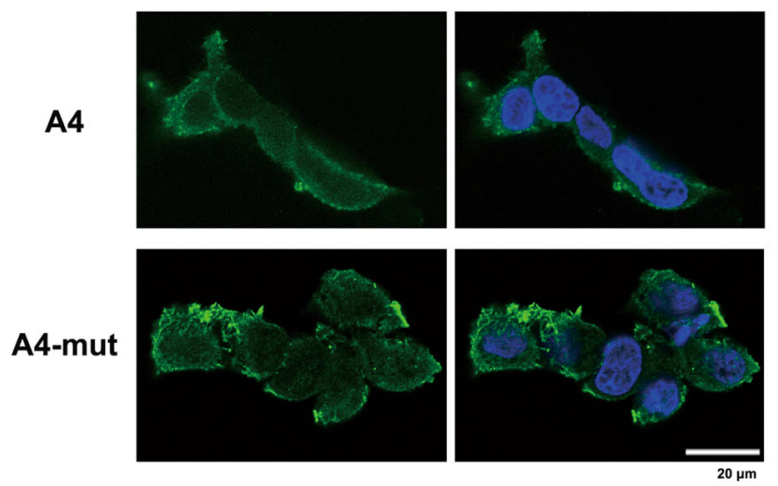

b

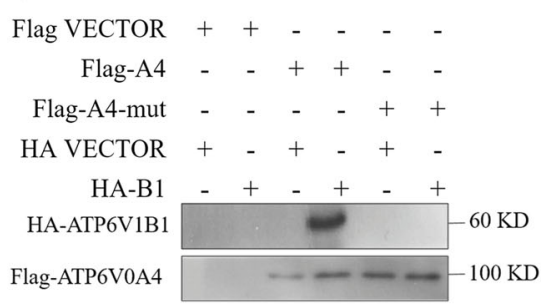

IP: anti-Flag

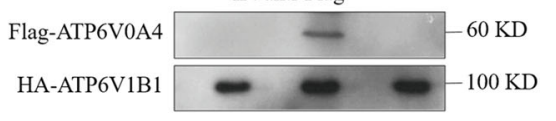

IP: anti-HA

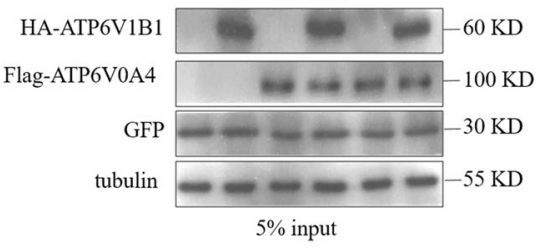

Fig. 3 Expression of WT and mutant ATP6VOA4 gene in HEK293T cells and interaction with B1 subunit. a Representative confocal images of pBOBi (empty-vector transfected) HEK293T cells (first row), or cells transiently transfected with A4 construct (second row), or A4-mut construct (third row). All panels show cells stained with anti-Flag antibodies (green). Nuclei are counter-stained with DAPI (blue). The results showed that both A4 and A4-mut are shown to be expressed on the cytoplasm, mostly on the cell membrane. The expression of A4-mut construct on the cell membrane increased, but the distribution was uneven. pBObi, empty vector control; A4, ATP6V0A4-wild type; A4-mut, ATP6V0A4/p.S544L mutant-type.

b Confirmation of the interaction effect between A4, A4-mut and B1 subunits by Co-IP. HEK293T cells were transfected with Flag vector, or Flag-A4, or Flag-A4-mut, or HA vector, or HA-B1. 5\% input was used as a positive control group, whole cell lysate was treated with tubulin antibody as internal reference and GFP antibody as external reference, and the loading and transfection effects were similar between the groups. Whole-cell lysate were separately immunoprecipitated with anti-FLAG antibody and anti-HA antibody. It showed that the expression levels between groups of ATP6V1B1 were comparable, and the expression levels of A4 and A4-mut were comparable. Subsequently, both positive and negative immunoprecipitates were blotted and probed with anti-Flag antibody or anti-HA antibody, and B1 and a4 subunits were detected to bind to each other. When ATP6VOA4 is mutated, B1 subunit cannot bind to mutated a4 subunit. Data shown are representative of three independent experiments. Flag-A4 Flag-tagged ATP6VOA4-wild typ, Flag-A4-mut Flag-tagged ATP6V0A4/p.S544L mutant-type, HA-B1 HA-tagged ATP6V1B1.

on the membrane (the a4 subunit is encoded by the ATP6VOA4 gene) affects the function of V-ATPase, thereby affecting the transport of cell membrane $\mathrm{H}^{+}$. To determine whether ATP6V0A4/p.S544L-mut affect the binding of the a4 subunit to the $\mathrm{B} 1$ subunit resulting in incorrect assembly of V-ATPase, an immunoprecipitation experiment was performed. Figure $3 \mathrm{~b}$ shows representative immunoblots loaded with immunoprecipitate fractions that were pulled down with anti-Flag antibody or anti-HA antibody from lysates of HEK293T cells transfected with Flag-A4, or Flag-A4-mut, or HA-B1 or empty vector control. Results showed that the expression levels of ATP6V1B1 among groups were comparable and the expression levels between ATP6VOA4-WT and -mut were comparable. Forward and reverse Co-IP were performed using anti-Flag or anti-HA antibodies. Results showed that a4 subunit-WT could bind to B1, while a4 subunit-mut could not bind to B1 subunit, which leads to incorrect assembly of the V1 and V0 domains, forming a structurally and functionally defective V-ATPase.
ATPase activity assay (NADH absorbance change A340) in HEK293T cells carrying ATP6VOA4-WT and p.S544L-mut

During the period in 5, 10, 15, $20 \mathrm{~min}, A T P 6 V 0 A 4 / \mathrm{p}$. S544L-mut cells showed relatively weak ATPase activity [e.g., ATP6VOA4-WT $(0.239 \pm 0.015 \mathrm{~A} / \mathrm{min})$ vs. p.S544Lmut $(0.091 \pm 0.012 \mathrm{~A} / \mathrm{min}), P<0.05]$. In the first $5 \mathrm{~min}, \mathrm{p}$. S544L-mut cells showed a decrease in slope compared with WT cells. Over time, the slope of the change in the absorbance of the ATPase NADH of p.S544L-mut cells was gradually consistent with that of WT cells (Fig. 4). The results show that the ATPase activity of p.S544L-mut carrier cells is weakened at an early stage. In order to maintain the overall cell function, it may be subsequently self-regulated and modified by endogenous pathways.

\section{PH fluorescence recovery assay in HEK293T cells carrying ATP6V0A4-WT and p.S544L-mut}

The recovery rate of $\mathrm{PH}$ in different time periods after rapid acidification of $\mathrm{NH}_{4} \mathrm{Cl}$ solution was compared (Fig. 5), and it was found that the p.S544-mut mutant could affect the hydrogen ion transport function of $\mathrm{V}$ - 


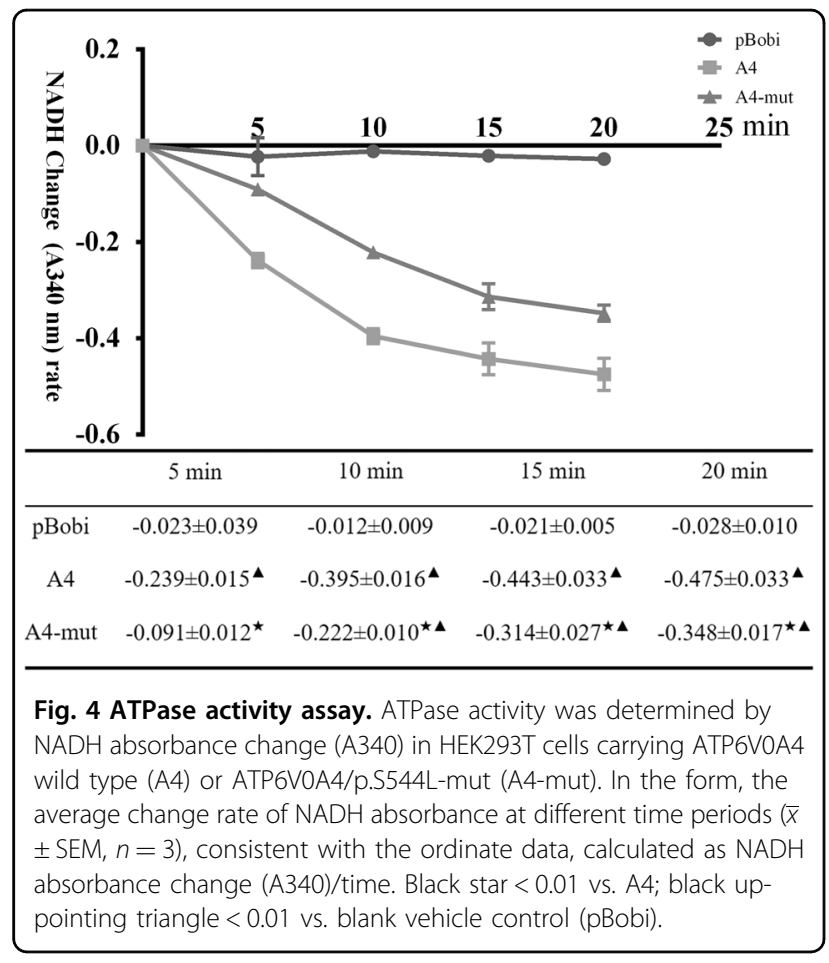

ATPase. In HEK293T cells transfected with ATP6VOA4WT, the cell pHi returned to 7.050 at $30 \mathrm{~min}$ after rapid acidification independent of sodium transport, and the average recovery rate of $\mathrm{pHi}$ was $(0.0384 \pm 0.0010) \mathrm{pH} \mathrm{U} /$ min. In HEK293T cells transfected with p.S544L-mut, the $\mathrm{pH}$ value returned to 6.967 after $30 \mathrm{~min}$ of rapid acidification without sodium transport, and the average recovery rate of $\mathrm{pHi}$ was $(0.0349 \pm 0.0001) \mathrm{pH} \mathrm{U} / \mathrm{min}$. The difference was not statistically significant $(P>0.05)$. In the $5,10,15$, and 25 min after adding $\mathrm{NH}_{4} \mathrm{Cl}$, the ATP6VOA4WT group showed significantly higher recovery rate than the p.S544L-mut group, which was $(0.1335 \pm 0.0060) \mathrm{pH}$ $\mathrm{U} / \mathrm{min}$ vs. $(0.0761 \pm 0.0015) \mathrm{pH} \mathrm{U} / \mathrm{min}$ in the first $5 \mathrm{~min}$; $(0.0740 \pm 0.0017) \mathrm{pH} \mathrm{U} / \mathrm{min}$ vs. $(0.0580 \pm 0.0008) \mathrm{pH} \mathrm{U} /$ $\mathrm{min}$ in the first $10 \mathrm{~min} ;(0.0617 \pm 0.0023) \mathrm{pH} \mathrm{U} / \mathrm{min}$ vs. $(0.0550 \pm 0.0017 \mathrm{pH} \mathrm{U} / \mathrm{min}$ in the first $15 \mathrm{~min} ;(0.0406 \pm$ $0.0016) \mathrm{pH} \mathrm{U} / \mathrm{min}$ vs. $(0.0370 \pm 0.0000) \mathrm{pH} \mathrm{U} / \mathrm{min}$ in the first $25 \mathrm{~min}(P<0.05)$, and after rapid acidification of the cells in the $\mathrm{NH}_{4} \mathrm{Cl}$ solution, the $\mathrm{pH}$ recovery rate gradually became uniform over time. As described in the previous experiments, it was demonstrated that p.S544L-mut carrier cells may reduce the difference in the rate of $\mathrm{PH}$ recovery by endogenous mechanisms in order to maintain the overall cell function.

\section{Discussion}

dRTA is a renal tubular disease with major defects in urinary acidification and acid excretion in the renal collecting duct system. The genetic form of dRTA has the characteristics of early onset age and serious disease,
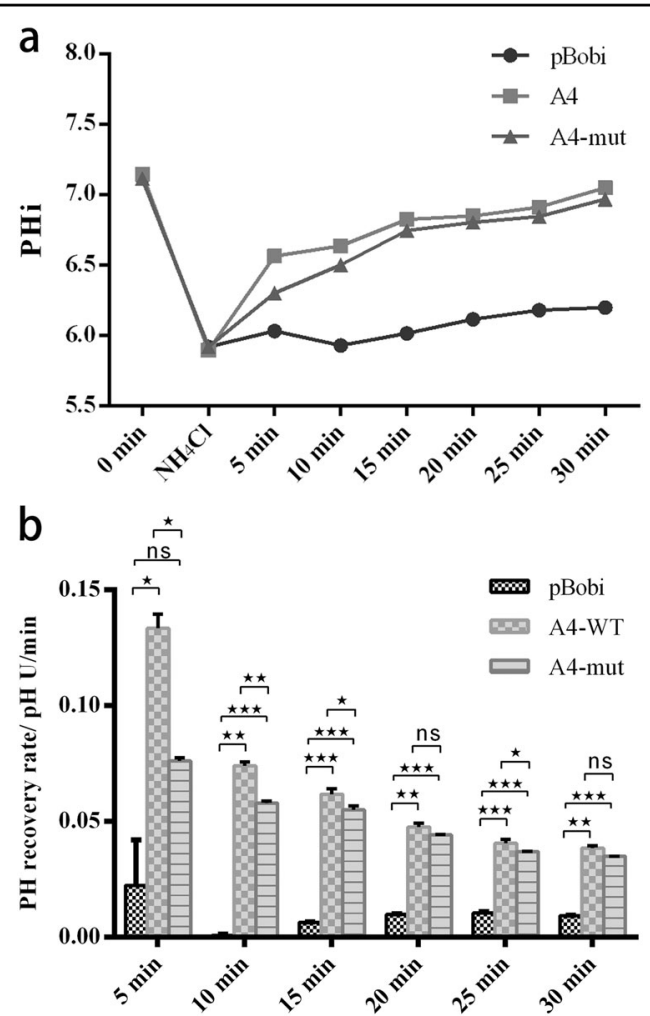

Fig. 5 PH fluorescence recovery assay in HEK293T cells carrying ATP6VOA4-WT and p.S544L-mut. a Trends in PH levels at different time points after rapid acidification of cells in $\mathrm{NH}_{4} \mathrm{Cl}$ solution. $\mathbf{b}$ In HEK293T cells, the PH recovery rates were compared at different times after $\mathrm{NH}_{4} \mathrm{Cl}$ solution rapidly acidified cells. ns, no significance; Black star $<0.05$, double black star $<0.01$, triple black star $<0.001$; pBObi empty-vector transfected, A4 ATP6VOA4-wild type, A4-mut ATP6VOA4/ p.S544L mutant-type. Error bars indicate \pm SEM.

which seriously endangers human health, and the clinical manifestations usually begin in infancy or childhood ${ }^{1,4,13}$. With the development of molecular biology theory and sequencing technology, a variety of genes related to hereditary dRTA and their mutations have been reported, including at least three different gene mutations: SLC4A1, ATP6V1B1, ATP6V0A4, encoding exchanger AE1 of $\mathrm{Cl}^{-}$/ $\mathrm{HCO}_{3}{ }^{-}, \mathrm{B} 1$ subunit and a4 subunit of V-ATPase, respectively ${ }^{4,14,15}$. More than 30 ATP6V1B1 mutations and 40 ATP6VOA4 mutations have been reported ${ }^{16,17}$, whereas China has only reported a few sporadic cases ${ }^{18,19}$. Mutations in SLC4A1 (AE1) are mainly inherited in an autosomal dominant manner, but also have autosomal recessive inheritance. The $A T P 6 V 0 A 4$ gene is located on chromosome 7q33-34 and has 23 exons, of which 20 exons encode a4 subunit containing 840 amino acids ${ }^{20}$. The ATP6V1B1 and ATP6VOA4 mutations affect two distinct subunits of V-ATPase: the B1 and a4 subunits, most of which are currently reported to be inherited in an autosomal recessive manner. However, patients with 
heterozygous ATP6V1B1 and ATP6VOA4 mutations, respectively had an increased risk of kidney stones and renal calcinosis in adulthood, or had incomplete $\mathrm{dRTA}^{21,22}$. Incomplete dRTA refers to insufficient uric acid in the renal collecting duct system of patients, but they usually have normal blood $\mathrm{pH}$ and bicarbonate, while acid provocation test reveals that uric acid deficiency does not cause urine $\mathrm{pH}$ below $5.3^{23}$. The patient with complete dRTA might exhibit mild clinical symptoms, such as mild metabolic acidosis or occasional kidney stones, or even severe clinical manifestations, such as severe metabolic acidosis, nephrocalcinosis, kidney stones, rickets, or osteomalacia, but preserved glomerular filtration rate. Except in the renal tissue, the B1 and a4 subunit genes of $\mathrm{V}$-ATPase are also expressed in the epithelial cells of the endolymphatic sac (ES) in the cochlea ${ }^{24}$, which could explain progressive sensorineural hearing loss or abnormally enlarged partial vestibular aqueduct in most patients $^{8,25-28}$. dRTA cases caused by ATP6V1B1 mutations might develop deafness during early stages, while dRTA cases caused by ATP6VOA4 mutations show mild or severe deafness or no deafness phenotype ${ }^{29,30}$. The difference in distribution and function of these two subunits may affect the heterogeneity of symptoms. Through genetic-phenotypic linkage analysis, we found that the family with p.S544L heterozygotes in the present study might be dominantly inherited, showing complete dRTA characteristics. Several studies have reported that several candidate genes are involved in hereditary dRTA, such as the Forkhead transcription factor Foxil. The ultrastructure of the distal nephron of the kidney of Foxi1 knockout mice was changed. In the $\alpha$-ICs of the collecting tube, the expression of various anion transporters, proton pumps and anion exchange proteins were deleted, accompanied by the deletion expression of Foxil in the inner ear and might be accompanied by sensorineural deafness $^{31}$. Another example is the deletion of other $\mathrm{V}$ ATPase subunits ${ }^{32}$, the chloride transporter SLC26A7 ${ }^{33}$, the $\mathrm{K}-\mathrm{Cl}$ co-transporter $\mathrm{KCC} 4^{34}$, hensin/DMBT1 ${ }^{35}$, Rhesus factor Rhcg ${ }^{36}$, etc.

The nephron cortical collecting duct (CCD) consists of chief cells that mediate $\mathrm{Na}^{+}, \mathrm{K}^{+}$, and water transport and ICs that are specifically used for acid-base transport, while ICs have two typical forms: acid-secreting $\alpha$-ICs and $\mathrm{HCO}_{3}{ }^{-}$secreting $\beta$-ICs. Chronic acidosis increases $\alpha$-ICs and downregulates $\beta$-ICs, thereby increasing the net acid secretion of $\mathrm{CCD}^{37}$. The hydrogen secretion function of the distal renal tubule is mainly accomplished by $\alpha$-ICs. In $\alpha$-ICs, under the action of intracellular carbonic anhydrase II (CA type II), $\mathrm{CO}_{2}$ combines with $\mathrm{H}_{2} \mathrm{O}$ to form $\mathrm{H}_{2} \mathrm{CO}_{3}$, which then dissociates to form $\mathrm{H}^{+}$and $\mathrm{HCO}_{3}{ }^{-}$. $\mathrm{H}^{+}$is transported to the small lumen by V-ATPase located in the luminal side membrane of the $\alpha$-ICs, while $\mathrm{HCO}_{3}{ }^{-}$is transported back to the blood by AE1 located in the basement membrane. The $\mathrm{H}^{+}$after secretion into the lumen combines with phosphate and $\mathrm{NH}_{3}$ in the lumen of the collecting duct ${ }^{38}$, and $\mathrm{H}^{+}$combines with $\mathrm{NH}_{3}$ in the lumen of the collecting duct to form $\mathrm{NH}_{4}{ }^{+}$. The actively reabsorbed $\mathrm{NH}_{4}{ }^{+}$dissociated to form $\mathrm{H}^{+}$and $\mathrm{NH}_{3}$, while $\mathrm{NH}_{3}$ was dispersed into the lumen and $\mathrm{H}^{+}$could be used as a substrate for V-ATPase. Therefore, the disorder of hydrogen secretion caused by mutations in the distal nephron may cause a decrease in the degree of acidification of the urine and a decrease in the secretion of $\mathrm{NH}_{4}{ }^{[+3}$. In addition, some transport factors are involved in the acid-secretion process of $\alpha-\mathrm{ICs}$, such as the $\mathrm{K}^{+}-\mathrm{Cl}^{-}$ cotransporter $\left(\mathrm{KCC}_{4}\right)$ found in the $\alpha$-ICs basolateral membrane of mice, where $\mathrm{Cl}^{-}$outflow maintains the normal function of AE1 and mutations encoding these protein genes could result in dRTA in mice ${ }^{8,34,39}$.

V-ATPase is an important member of the ATP enzyme family. V-ATPase transfer protons relying on the energy produced by hydrolyzing ATP, which produces an electrochemical gradient across the membrane and regulates the $\mathrm{pH}$ inside and outside the cell. VATPase has played an important role in receptormediated endocytosis, protein degradation, storage of secreted proteins, intracellular membrane trafficking, absorption of $\mathrm{Na}^{+}$, renal acidification, bone resorption of osteoclasts, maturation of male sperm, etc. Many human diseases such as RTA, osteopetrosis, and tumor metastasis have important relationships with the physiological functions of V-ATPase ${ }^{40}$. V-ATPase consists of two functional parts, V1 and V0. The water-soluble spherical V1 is exposed on the surface of the membrane and has the function of catalyzing the hydrolysis of ATP and consists of eight subunits of $\mathrm{A}-\mathrm{H}$. The fat-soluble $\mathrm{V} 0$ is embedded in the membrane and is responsible for the transfer of protons and are composed of six subunits a, c, c', c', d, and e. The hexamer A3B3 composed of the A subunit and the $B$ subunit in the V1 part is an energizing part, and it is connected to a peripheral rod (composed of C, E, G, H, a, and e subunits) to form a stator structure. The lipoprotein structure consisting of the subunits c, c', and c" in the V0 moiety forms a rotor structure with the central rod (composed of subunits $d$, $\mathrm{D}$, and $\mathrm{F})^{41,42}$. The interaction between the subunits and the correct assembly are the key to maintaining the function of the V-ATP enzyme. For example, two proton hemichannels were formed inside a subunit towards the cytoplasm and the organelle cyst. When a proton in the cytoplasm enters the first proton hemichannel, it binds to a negatively charged glutamic acid residue on $\mathrm{c}$, c', or c", while V1 decomposes the energy generated by ATP to drive the D and F subunit rotation. Then, the lipoprotein cyclic structure formed by c, c', and c" is rotated. A neutral glutamic acid residue rotates with the lipoprotein loop, and when glutamate is rotated to 
another proton hemichannel, an arginine residue (R735) on the a subunit is interacts with the glutamate residue, lowering its $\mathrm{pKa}$ value to release protons, and the protons reach the other side of the membrane through the second proton hemichannel ${ }^{43,44}$. The a4 subunit may be involved in the assembly of V-ATPase, as well as the activity of ATPase and the transport of ions ${ }^{45,46}$. The a4 and $\mathrm{B} 1$ are subunit isoforms that occur together in the kidney ${ }^{47}$, both $A T P 6 V 1 B 1$ and ATP6VOA4 mutations can cause dRTA. The mutations cause structural abnormalities in B1 or a4 may affect the binding of their two subunits, which in turn may hinder the correct assembly of V-ATPase. The free subunits may be localized to the brush border membrane, but the assembled $\mathrm{V}$-ATPase is not sufficiently active, thereby reducing the ability of $\alpha$-ICs to pump $\mathrm{H}^{+}$into the lumen, leading to the development of $\mathrm{dRTA}^{48}$. Another example is the site at the carboxy terminus of a 4 that binds to the glycolytic enzyme-phosphofructokinase, and mutations at this site could lead to dRTA ${ }^{46}$. In vitro experiments, cycloheximide chase assays revealed that V-ATPase a4 $R 449 H$ were unstable relative to wildtype. Moreover, the a4 $R 449 H$ variant of dRTA remained in the endoplasmic reticulum, and the cell surface expression was defective. a4 $\mathrm{R} 449 \mathrm{H}$ increased the association with the V0 assembly factor VMA21 and decreased association with ATP6V1B $1^{49}$. In addition, mutations in ATP6V0A4 impair V-ATPase function, causing renal urinary acidosis, leading to bone softening due to loss of bone minerals (demineralization) and other causes. V-ATPase on the plasma membrane of osteoclasts plays an important role in bone resorption. Osteoclasts form a closed space around bone cells, and osteoclast VATPase pumps protons to the closed space to make the space an acidic environment, which causes the bone matrix to dissolve, activates secreted proteases, and promotes bone resorption ${ }^{50}$. Gene defects in V-ATPase in osteoclasts could also lead to recessive genetic disease such as osteopetrosis ${ }^{51,52}$.

This dRTA family has a distinctive feature, which is the incidence of five dRTA patients in the family. Mutation-linkage analysis suggests that the family has dominant inheritance characteristics. To our knowledge, the clinical features of human heterozygous ATP6VOA4 mutants have not been well described to date, but in wild/ATP6VOA4 deletion hybrid mice, high chloride metabolic acidosis has been demonstrated under chronic acid loading conditions ${ }^{53}$. In addition, ATP6V0A4 p.S544L heterozygotes were found in a 40 year-old male patient with hypokalemic periodic paralysis, renal calcification, and alkaline urine incomplete dRTA confirmed by the $\mathrm{NH} 4 \mathrm{Cl}$ load test, the furosemide-fludrocortisone loading test, and the $\mathrm{HCO}_{3}{ }^{-}$loading test in Japanese population ${ }^{21}$, which is consistent with our findings in Chinese Han population. However, some patients in this study showed more severe complete dRTA. We also found no deafness phenotype among members of the family. These features and previous literature may indicate that the ATP6VOA4 gene acts either dominantly or with recessive inheritance.

\section{Acknowledgements \\ This work was supported by the science and technology project of Fujian Province, China (No. 2018Y0012), National Natural Science Foundation of China (No. 81874379), and Financial scheme for young talents training program of Fujian Health industry (No. 2017-ZQN-1)

\begin{abstract}
Author details
${ }^{1}$ Shengli Clinical Medical College of Fujian Medical University, Fuzhou 350001, China. ${ }^{2}$ Department of Traditional Chinese Medicine, Fujian Provincial Hospital, Fuzhou 350001, China. ${ }^{3}$ Department of Traditional Chinese Medicine, the First Affiliated Hospital, Fujian Medical University, Fuzhou 350005, China.

${ }^{4}$ Department of Paediatrics, Fujian Provincial Hospital, Fuzhou 350001, China. ${ }^{5}$ Department of Interventional Radiology, Fujian Provincial Hospital, Fuzhou 350001, China
\end{abstract}

Conflict of interest

The authors declare that they have no conflict of interest.

\section{Publisher's note}

Springer Nature remains neutral with regard to jurisdictional claims in published maps and institutional affiliations.

Received: 20 December 2019 Revised: 11 February 2020 Accepted: 11 February 2020

Published online: 02 March 2020

\section{References}

1. Yaxley, J. \& Pirrone, C. Review of the diagnostic evaluation of renal tubular acidosis. Ochsner J. 16, 525-530 (2016).

2. Trepiccione, F. et al. New findings on the pathogenesis of distal renal tubular acidosis. Kidney Dis. 3, 98-105 (2017).

3. Vasquez-Rios, G., Westrich, D. J. Jr., Philip, I., Edwards, J. C. \& Shieh, S. Distal renal tubular acidosis and severe hypokalemia: a case report and review of the literature. J. Med. Case Rep. 13, 103 (2019).

4. Both, T. et al. Everything you need to know about distal renal tubular acidosis in autoimmune disease. Rheumatol. Int. 34, 1037-1045 (2014).

5. Kitterer, D., Schwab, M., Alscher, M. D., Braun, N. \& Latus, J. Drug-induced acidbase disorders. Pediatr. Nephrol. 30, 1407-1423 (2015).

6. Jung, S. W. et al. Renal tubular acidosis in patients with primary Sjogren's syndrome. Electrolyte Blood Press. 15, 17-22 (2017).

7. Soares, S. B. M., de Menezes Silva, L. A. W., de Carvalho Mrad, F. C. \& Simoes, E. S. A. C. Distal renal tubular acidosis: genetic causes and management. World J Pediatr. https://doi.org/10.1007/s12519-019-00260-4 (2019).

8. Mohebbi, N. \& Wagner, C. A. Pathophysiology, diagnosis and treatment of inherited distal renal tubular acidosis. J. Nephrol. 31, 511-522 (2018).

9. Fry, A. C. \& Karet, F. E. Inherited renal acidoses. Physiology 22, 202-211 (2007).

10. Wang, K., Li, M. \& Hakonarson, H. ANNOVAR: functional annotation of genetic variants from high-throughput sequencing data. Nucleic Acids Res. 38, e164 (2010).

11. Richards, S. et al. Standards and guidelines for the interpretation of sequence variants: a joint consensus recommendation of the American College of Medical Genetics and Genomics and the Association for Molecular Pathology. Genet Med. 17, 405-424 (2015).

12. Van Itallie, C. M. \& Anderson, J. M. Phosphorylation of tight junction transmembrane proteins: many sites, much to do. Tissue Barriers 6, e1382671 (2018).

13. Mustaqeem, R. \& Arif, A. Renal Tubular Acidosis. (StatPearls, 2019). 
14. Batlle, D. \& Haque, S. K. Genetic causes and mechanisms of distal renal tubular acidosis. Nephrol. Dial. Transpl. 27, 3691-3704 (2012).

15. Santos, F., Gil-Pena, H. \& Alvarez-Alvarez, S. Renal tubular acidosis. Curr. Opin. Pediatr. 29, 206-210 (2017).

16. Boualla, L., Jdioui, W., Soulami, K., Ratbi, I. \& Sefiani, A. Clinical and molecular findings in three Moroccan families with distal renal tubular acidosis and deafness: report of a novel mutation of ATP6V1B1 gene. Curr. Res. Transl. Med. 64, 5-8 (2016).

17. Shine, L., Kilty, C., Gross, J. \& Kennedy, B. Vacuolar ATPases and their role in vision. Adv. Exp. Med. Biol. 801, 97-103 (2014).

18. Shao, L. et al. A novel SLC4A1 variant in an autosomal dominant distal renal tubular acidosis family with a severe phenotype. Endocrine 37, 473-478 (2010).

19. Zhang, C. et al. Clinical evaluation of Chinese patients with primary distal renal tubular acidosis. Intern. Med. 54, 725-730 (2015).

20. Smith, A. N. et al. Mutations in ATP6N1B, encoding a new kidney vacuolar proton pump 116-kD subunit, cause recessive distal renal tubular acidosis with preserved hearing. Nat. Genet. 26, 71-75 (2000).

21. Imai, E. et al. A novel heterozygous mutation in the ATP6VOA4 gene encoding the V-ATPase a4 subunit in an adult patient with incomplete distal renal tubular acidosis. Clin. Kidney J. 9, 424-428 (2016).

22. Zhang, J. et al. Incomplete distal renal tubular acidosis from a heterozygous mutation of the V-ATPase B1 subunit. Am. J. Physiol. Ren. Physiol. 307, F1063-F1071 (2014).

23. Wrong, O. \& Davies, H. E. The excretion of acid in renal disease. Q. J. Med. 28, 259-313 (1959).

24. Dou, H. et al. Co-expression of pendrin, vacuolar H+-ATPase alpha4-subunit and carbonic anhydrase II in epithelial cells of the murine endolymphatic sac. J. Histochem. Cytochem. 52, 1377-1384 (2004).

25. Besouw, M. T. P. et al. Clinical and molecular aspects of distal renal tubular acidosis in children. Pediatr. Nephrol. 32, 987-996 (2017).

26. Lorente-Canovas, B. et al. Mice deficient in H+-ATPase a4 subunit have severe hearing impairment associated with enlarged endolymphatic compartments within the inner ear. Dis. Model. Mech. 6, 434-442 (2013).

27. Mohebbi, N. et al. Homozygous and compound heterozygous mutations in the ATP6V1B1 gene in patients with renal tubular acidosis and sensorineural hearing loss. Clin. Genet. 83, 274-278 (2013).

28. Stover, E. H. et al. Novel ATP6V1B1 and ATP6V0A4 mutations in autosomal recessive distal renal tubular acidosis with new evidence for hearing loss. J. Med. Genet. 39, 796-803 (2002)

29. Breton, S. \& Brown, D. Regulation of luminal acidification by the V-ATPase. Physiology 28, 318-329 (2013).

30. Gao, Y. et al. Mutation analysis and audiologic assessment in six Chinese children with primary distal renal tubular acidosis. Ren. Fail. 36, 1226-1232 (2014).

31. Blomqvist, S. R. et al. Distal renal tubular acidosis in mice that lack the forkhead transcription factor Foxi1. J. Clin. Invest. 113, 1560-1570 (2004).

32. Smith, A. N., Borthwick, K. J. \& Karet, F. E. Molecular cloning and characterization of novel tissue-specific isoforms of the human vacuolar $\mathrm{H}(+)$-ATPase $\mathrm{C}$, $G$ and $d$ subunits, and their evaluation in autosomal recessive distal renal tubular acidosis. Gene 297, 169-177 (2002).

33. $\mathrm{Xu}$, J. et al. Deletion of the chloride transporter slc26a7 causes distal renal tubular acidosis and impairs gastric acid secretion. J. Biol. Chem. 284 29470-29479 (2009).

34. Boettger, T. et al. Deafness and renal tubular acidosis in mice lacking the $\mathrm{K}-\mathrm{Cl}$ co-transporter Kcc4. Nature 416, 874-878 (2002).
35. Gao, X. et al. Deletion of hensin/DMBT1 blocks conversion of beta- to alphaintercalated cells and induces distal renal tubular acidosis. Proc. Natl Acad. Sci. USA 107, 21872-21877 (2010).

36. Biver, S. et al. A role for Rhesus factor Rhcg in renal ammonium excretion and male fertility. Nature 456, 339-343 (2008).

37. Schwartz, G. J. et al. SDF1 induction by acidosis from principal cells regulates intercalated cell subtype distribution. J. Clin. Invest. 125, 4365-4374 (2015).

38. Laing, C. M., Toye, A. M., Capasso, G. \& Unwin, R. J. Renal tubular acidosis: developments in our understanding of the molecular basis. Int J. Biochem. Cell Biol. 37, 1151-1161 (2005).

39. Kobayashi, K., Uchida, S., Mizutani, S., Sasaki, S. \& Marumo, F. Developmental expression of CLC-K1 in the postnatal rat kidney. Histochem. Cell Biol. 116, 49-56 (2001)

40. Wilkens, S, Zhang, Z, \& Zheng, Y. A structural model of the vacuolar ATPase from transmission electron microscopy. Micron 36, 109-126 (2005).

41. Forgac, M. Vacuolar ATPases: rotary proton pumps in physiology and pathophysiology. Nat. Rev. Mol. Cell Biol. 8, 917-929 (2007).

42. Schumacher, K. \& Krebs, M. The V-ATPase: small cargo, large effects. Curr. Opin Plant Biol. 13, 724-730 (2010).

43. Blake-Palmer, K. G., Su, Y., Smith, A. N. \& Karet, F. E. Molecular cloning and characterization of a novel form of the human vacuolar H+-ATPase e-subunit: an essential proton pump component. Gene 393, 94-100 (2007).

44. Duarte, A. M. et al. Structure and localization of an essential transmembrane segment of the proton translocation channel of yeast H+-V-ATPase. Biochim. Biophys. Acta 1768, 218-227 (2007).

45. Kawasaki-Nishi, S., Nishi, T. \& Forgac, M. Arg-735 of the 100-kDa subunit a of the yeast V-ATPase is essential for proton translocation. Proc. Natl Acad. Sci. USA 98, 12397-12402 (2001)

46. Su, Y. et al. Human H+ATPase a4 subunit mutations causing renal tubular acidosis reveal a role for interaction with phosphofructokinase-1. Am. J. Physiol. Ren. Physiol. 295, F950-F958 (2008).

47. Toei, M., Toei, S. \& Forgac, M. Definition of membrane topology and identification of residues important for transport in subunit a of the vacuolar ATPase. J. Biol. Chem. 286, 35176-35186 (2011).

48. Yang, Q., Li, G., Singh, S. K., Alexander, E. A. \& Schwartz, J. H. Vacuolar H+ -ATPase B1 subunit mutations that cause inherited distal renal tubular acidosis affect proton pump assembly and trafficking in inner medullary collecting duct cells. J. Am. Soc. Nephrol. 17, 1858-1866 (2006).

49. Esmail, S. et al. Molecular mechanisms of cutis laxa- and distal renal tubular acidosis-causing mutations in V-ATPase a subunits, ATP6V0A2 and ATP6V0A4 J. Biol. Chem. 293, 2787-2800 (2018).

50. Toyomura, T. et al. From lysosomes to the plasma membrane: localization of vacuolar-type $\mathrm{H}+$-ATPase with the a3 isoform during osteoclast differentiation. J. Biol. Chem. 278, 22023-22030 (2003).

51. Henriksen, $\mathrm{K}$. et al. Dissociation of bone resorption and bone formation in adult mice with a non-functional $V$-ATPase in osteoclasts leads to increased bone strength. PLOS ONE 6, e27482 (2011).

52. Kartner, N. et al. Inhibition of osteoclast bone resorption by disrupting vacuolar H+-ATPase a3-B2 subunit interaction. J. Biol. Chem. 285 37476-37490 (2010).

53. Norgett, E. E. et al. Atp6v0a4 knockout mouse is a model of distal renal tubular acidosis with hearing loss, with additional extrarenal phenotype. Proc. Natl Acad. Sci. USA 109, 13775-13780 (2012). 